\title{
Mediamorfosis del Cine Documental: Reflexiones en torno a las fronteras de un género en expansión
}

\author{
Nazly López Díaz \\ Investigadora junior Minciencias Colombia \\ nazlymaryiths@gmail.com \\ https://orcid.org/0000-0003-0111-1055
}

\section{Mediamorphosis of Documentary Film: Reflections on the frontiers of an expanding genre}

\begin{abstract}
RESUMEN ABSTRACT
La evolución del cine documental desde su surgimiento y en particular a partir de la era digital, conduce a preguntarse por las fronteras de un género cuya evolución narrativa ha estado marcada por los avances tecnológicos y el punto de vista del autor respecto a la realidad documentada. En el escenario de la convergencia y el ecosistema de medios, las

nuevas formas de consumo, apropiación y producción de contenido retan a asumir formas distintas de interacción con las audiencias, instándolas a comprometerse con las problemáticas tratadas. El lugar de la enunciación deja de estar a un solo lado de la cámara, para hacerse presente en ambos ejes. Así, la aparición del otro en la ecuación narrativa permite que el cine documental encuentre un terreno propicio para seguir cumpliendo su misión profética, a través de formatos no convencionales como el documental para la Web, las narrativas gamificadas y las experiencias inmersivas en

The evolution of documentary film since its birth and especially in the digital era, leads us to wonder about the limits of a genre whose evolution of language and narratives, has been determined by technological advances and the author point of view regarding the documented reality. In the scenario of convergence and the media ecosystem, the new forms of consumption, appropriation and production of content, challenge documentary films to assume different ways of interaction with audiences, pushing them to take committed positions in relation to the issues addressed. The place of enunciation is now on both sides of the camera axis. In this order of ideas, the appearance of the other in the narrative equation, allows documentary film to find a fertile field to continue fulfilling its prophetic mission, through non-conventional formats such as Webdoc, gamified narratives and VR immersive experiences.
\end{abstract} realidad virtual.

PALABRAS CLAVE

KEYWORDS

Cine documental; Nuevas narrativas; Convergencia mediática; Mediamorfosis.

Documentary film; New narratives; Media convergence; Mediamorphosis. Hipertext.net, (23), 21-30. https://doi.org/10.31009/hipertext.net.2021.i23.03 


\section{Mediamorfosi del Cinema Documental: Reflexions sobre les fronteres d'un gènere en expansió}

\section{RESUM}

L'evolució del cinema documental des del seu sorgiment i en particular a partir de l'era digital porta a preguntar-se per les fronteres d'aquest gènere, que ha tingut una evolució narrativa marcada pels avenços tecnològics i per el punt de vista de l'autor respecte a la realitat documentada. En un escenari de convergència en l'ecosistema de mitjans, les noves formes de consum, apropiació i producció de contingut repten a assumir formes diferents d'interacció amb les audiències, instant-les a comprometre's amb les problemàtiques tractades. El lloc de l'enunciació deixa d'estar a un sol costat de la càmera i es fa present a tots dos eixos.Així, l'aparició de l'altre en l'equació narrativa permet que el cinema documental trobi un terreny propici per a seguir complint la seva missió profètica, a través de formats no convencionals com el documental Web, les narratives gamificades i les experiències immersives en realitat virtual.

\section{PARAULES CLAU}

Cinema documental; Noves narratives; Convergència mediàtica; Mediamorfosi.

\section{Introducción}

Partiendo de la perspectiva clásica del cine documental, este trabajo realiza un recorrido histórico desde los albores del siglo XX hasta la era digital, para analizar la forma en que la técnica transforma el lenguaje y lo adapta al medio de difusión, en una evolución permanente, que permite hoy en día cuestionar los límites entre géneros, e indagar en un hecho sin precedentes, por los consumos, los consumidores y las ventanas alternas de difusión del documental contemporáneo. En la misma línea, el nuevo estado de cosas obliga a la redefinición de la autoría, es decir, del lugar del director de documentales dentro del relato, quien hoy en día abandona su posición omnipotente, para devenir en diseñador de experiencias y creador de estrategias de interacción con los usuarios.

De este modo, el cine documental dentro de la llamada convergencia de medios, atraviesa su propia Mediamorfosis, para transformarse en un producto que se expande a través de diferentes plataformas, y más que contar historias, genera experiencias. En este movimiento el documental se cruza con distintos lenguajes y tecnologías, que lo llevan a trascender sus propios límites fundacionales, para hallar en otras formas expresivas como el documental para la Web, los documentales gamificados y el documental en realidad virtual, posibles caminos para amplificar su mensaje.

\section{Marco teórico}

Fidler (1998), describe el concepto de Mediamorfosis como las transformaciones que sufre un medio de comunicación ya establecido, en su proceso de adaptación a la tecnología y los cambios culturales de una época. Es un proceso evolutivo, en la medida en que toda forma moderna tiene un precedente. Como señala el autor:

Este continuo de transformaciones y adaptaciones, como veremos, es en realidad un proceso complejo, comparable, en muchos sentidos, a la evolución de las especies. Las formas exitosas de nuevos medios, al igual que las nuevas especies, no emergen espontáneamente, de la nada, todas requirieron vínculos con el pasado. (Fidler, 1998)

En este punto la teoría de la Mediamorfosis como un ejercicio de evolución individual, se encuentra con la teoría del Ecosistema de medios, que es entendido como un sistema complejo que a semejanza de un ecosistema biológico, involucra para su equilibrio la participación conjunta e interacción de las especies, en este caso de seres humanos, medios de comunicación y tecnología:

Podemos afirmar que el estudio ecológico de los medios de comunicación no se puede restringir a los medios de comunicación en sentido estricto, sino que también debe incluir la tecnología y el lenguaje y las interacciones de 
estos tres ámbitos, que juntas forman un ecosistema de medios. (Logan, 2010, p. 33-34)

Estas interacciones en el plano real, trascienden el mero intercambio objetivo y convergen posibilitando la emergencia de relaciones en el terreno simbólico. En ese sentido y siguiendo a Jenkins (2008), el concepto de convergencia más que a un proceso tecnológico consistente en el flujo de información asequible e intercambiable, que se propaga a través del ecosistema mediático, alude a un cambio cultural en el modo en que el consumo deja de ser pasivo y se plantea la posibilidad de acceder a la información de formas variadas. Esto permite a los usuarios establecer y conformar a su gusto, las redes que les permiten acceder a sus intereses. En ese sentido y como manifiesta Lovato (2015), "la convergencia se produce en el cerebro de los consumidores, mediante sus interacciones tecno-sociales" (p. 35).

Para Fidler (1998) la convergencia no es un fenómeno nuevo, sino parte constitutiva de la Mediamorfosis. Dicha convergencia se produce a gran escala, en el momento en que confluyen los grandes medios y en pequeña escala, a nivel de las interacciones del día a día que modelan, si se quiere, la relación con el medio y con la tecnología. El autor aclara que más que fusiones, "la convergencia implica la transformación de cada ente convergente, así como la creación de nuevos entes" (p. 63).

Pero más allá del fenómeno del medio en sí y su transformación, vale la pena preguntarse qué pasa con el contenido y cómo esto afecta lo que entendemos por género dentro de un lenguaje comunicativo. Fidler (1998) menciona que "los rasgos característicos de los medios se corporizan y se transmiten a través de códigos comunicativos que llamamos lenguajes" (p. 59), estos últimos son los que a la luz de Altman (2000), terminan contribuyendo a configurar las claves de lo que se denomina género en el ámbito del cine.

Altman (2000), más que una clasificación taxativa aplicada a las historias del cine, considera que los géneros tienen que ver tanto con las lógicas de la cadena productiva, como con la coexistencia de unas claves textuales que entran en diálogo con las audiencias. Entre las particularidades a observar para comprender los géneros cinematográficos, el autor menciona los procesos industrializados de producción del cine, las estructuras sobre las cuales se construyen los relatos, los intereses de los distribuidores en cuanto al modo como los contenidos son comercializados y finalmente una postura de identificación, que tiene que ver con el modo como el contenido es recibido por el público.

El cine documental puede considerarse un género en tanto se ajusta a las condiciones mencionadas por Altman (2000), ya que tiene una forma productiva con sus características propias, una lógica narrativa interna, se distribuye en circuitos especializados y en general el público puede clasificar y enten- der su contenido dentro de la misma categoría. Sin embargo, lo que es innegable y comúnmente aceptado en el documental clásico y el lineal, comienza desdibujarse con la incorporación de las nuevas narrativas derivadas de los medios contemporáneos.

En este panorama surge como un reto para el documental lineal, el concepto de Narrativa transmedia, que alude a las formas de narración que surgen en medio del nuevo ecosistema mediático, y de la exploración de los cruces entre los lenguajes característicos de los distintos medios (digitales y análogos), con el objetivo de construir un universo narrativo: "Se trata, de tal modo, de una elaboración semiótica compleja y elástica, que utiliza las cualidades de los distintos soportes para avanzar en el relato" (Valdettaro, 2015, p. 73).

En la forma ideal de la narración transmediática, cada medio hace lo que se le da mejor, de suerte que una historia puede presentarse en una película y difundirse a través de la televisión, las novelas y los cómics; su mundo puede explorarse en videojuegos o experimentarse en un parque de atracciones. Cada entrada a la franquicia ha de ser independiente, de forma que no sea preciso haber visto la película para disfrutar con el videojuego y viceversa. (Jenkins, 2008, p. 101-102)

Para Irigaray (2015) un rasgo fundamental del transmedia, adicional a su tránsito entre plataformas, es la posibilidad de generar narrativas participativas, de modo que los usuarios entren a hacer parte de la trama tanto en los territorios virtuales, como en los escenarios reales que convoca la historia. El documental lineal, que partiera como un lenguaje unívoco y teledirigido, entra en otro tipo de diálogo con la audiencia, cuando aparecen los usuarios en la ecuación. En dicho contexto, estos últimos se convierten en prosumidores, es decir aquellos que dejaron de ser consumidores pasivos, para formar parte de los relatos, mediante los contenidos que crean y comparten.

los documentales continuarán emitiéndose de manera lineal en los medios tradicionales (cine y televisión), en el ámbito de las nuevas tecnologías empiezan a concebirse como proyectos en permanente construcción que, a través de la convergencia mediática y de la participación de los usuarios, podría brindarle un mayor ciclo de vida y nuevas perspectivas a este nuevo formato. (Rosique, 2015, p. 66)

Contrario a lo que se pensaba, que la emergencia de un medio conduciría a la desaparición de su antecesor, la historia ha demostrado la posible coexistencia de los distintos medios, como representantes de las tecnologías que permitieron su desarrollo. En el mismo sentido, el mudar de características para sobrevivir, tal cual lo hacen las especies, termina siendo la esencia de la Mediamorfosis del cine documental: un continuo trasegar de las formas de comunicación y los medios, para permanecer vigentes en un sistema cambiante (Fidler, 1998). 


\section{Metodología}

La presente es una aproximación de tipo teórico que propone un recorrido por la historia del cine documental, en paralelo con las principales innovaciones tecnológicas que lo influenciaron, para comprender su proceso de evolución en el tiempo. En ese sentido, el trabajo se plantea como base teórica para futuras exploraciones que permitan, mediante la aplicación de herramientas de análisis descriptivas y exploratorias, expandir el alcance del análisis de los derroteros del género documental, en el marco de la Mediamorfosis.

\section{Discusión}

\subsection{Inmersión profunda en la historia del cine documental}

El cine tuvo cabida entre los primeros espectadores, gracias al campo que la fotografía, el teatro y algunos juguetes pre cinematográficos le abrieron (Fidler, 1998). Sin embargo, para este análisis avanzamos en la historia hasta el punto en el que el cine documental surge y es reconocido como tal, hablamos de los años veinte.

Entre las revisiones teóricas que se le han hecho al cine documental, destaca la aproximación de Erik Barnouw (2006), quien aborda el género desde una perspectiva histórica, ubicando según el momento del siglo $X X$, tendencias en las formas de narrar. Un ejemplo de esto sería la prevalencia de la voz en off como guía del relato, o la sustracción de la narración para dar protagonismo a las imágenes. Dichas tendencias se relacionan con el discurso enunciado y las intenciones del autor, pues modelan la percepción del espectador con respecto al tema tratado.

Estos modos de narrar, están a su vez relacionados con las innovaciones técnicas propias de cada época, como por ejemplo la aparición del sonido y de dispositivos portátiles y ligeros, por lo que podría afirmarse que el lenguaje documental, ha estado en relación directa con la manera como los equipos, los medios y las formas de comunicación se transforman, es decir con el fenómeno mediamórfico.

Desde los primeros registros obtenidos por los emisarios de las casas cinematográficas, hasta la aparición del sonido, la cámara de cine fue la única herramienta con la que contaron los pioneros. Si bien el registro de la realidad en las primeras películas recurre a la puesta en escena, como sucede en Nanook el esquimal (1922) de Robert Flaherty, y la recordada secuencia del iglú seccionado, que permite atisbar la vida íntima de esta familia Inuit, la cuestión que salta a la vista, es el hecho que el aparato con su volumen y peso, operaba como una obstrucción para acceder al mundo histórico sin resultar invasivo. Es por este motivo que la relación del cineasta con respecto a su objeto filmado, dista por aquellos años de cerca- nía e intimidad y desde luego, descarta cualquier oportunidad de participación por parte del espectador.

Se sabe que Jean Vigo experimentó en A Propósito de Niza (1929), ocultando la cámara y apostándola en una silla de ruedas desde donde era operada, para lograr esos trávelin callejeros que la hacían imperceptible a la mirada de los incautos (Barnouw, 2006). En este caso la estrategia del director consistió en ocultar el dispositivo, para captar el gesto oculto de aquella "sociedad abandonada así misma" (Vigo, 1989, p. 138).

Sin embargo y pese a los trucajes, la presencia de la cámara continuó siendo una barrera, que separaba tajantemente a quienes estaban de uno y otro lado de la lente. Esto aplicaba tanto en referencia a la relación con el espectador, como al lugar desde donde se producía el discurso. No es un secreto que en sus primeros años el cine estuvo relacionado con las élites y en muchos casos fue subvencionado por el Estado y empleado como herramienta de propaganda (Barnouw, 2006). Por esta razón y por una mirada etnocéntrica también imperante, parte del cine hecho durante el siglo XX registró a los otros, desde una perspectiva exótica y extrañada. La imagen construida, buena o mala, determinó incluso el destino de grupos humanos, ratificando que quien enuncia el discurso, termina teniendo una relación hegemónica sobre aquellos de quienes habla.

La aparición del sonido más que un testimonio cercano, trajo aparejada la voz del narrador, esa que se dirigía al espectador en tono de autoridad, para explicarle el mundo que se representaba ante sus ojos (Nichols, 1991). Este cine ligado principalmente a la propaganda y producido con ocasión del enfrentamiento de los bandos en la Segunda Guerra Mundial, continuaba ubicando a los sujetos filmados en la tercera persona. Ellos eran los otros, esos diferentes susceptibles de ser analizados, evaluados, medidos, tal como puede apreciarse en obras como Las Hurdes Tierra sin pan (1933) de Luis Buñuel y la serie de Frank Capra Why we fight (1942), sobre los motivos que justificaron la entrada de los Estados Unidos a la guerra.

Justamente la necesidad de informar desde el frente de batalla, expuso una serie de dificultades técnicas del equipo utilizado hasta el momento. De este modo se desarrollaron cámaras más ligeras y más tarde, aparece la grabadora de sonido magnético, que abrió la posibilidad al cine de lo real, de poder sincronizar la imagen con el sonido (Rabiger, 2001).

Así el cine documental posterior a la guerra, rompe con la tendencia de retratar grandes colectivos y entra en la cotidianidad de los individuos, para indagar por cuestiones más existenciales como la noción de felicidad, tal como sucede en Crónica de un Verano (1961) de Jean Rouch y Edgar Morin. La película que parte como un experimento comunicativo y deviene en un maravilloso manual de realización documental, es represen- 
tante del Cinema verité, movimiento estético y metodológico que apunta a catalizar situaciones ante la cámara, generando un ejercicio interactivo entre el realizador y los sujetos filmados. Por oposición al verité, el cine directo americano surgido durante el mismo periodo, se decantó por la no intervención y por dejar que la vida sucediera delante de la cámara (Barnouw, 2006). En uno y en otro caso, la aparición de un equipo ligero y portátil, favoreció la incursión de los cineastas en la intimidad de las personas.

En el caso latinoamericano según Paranaguá (2003), el documental estuvo ligado en principio al cine de institutos avalado por los gobiernos, por lo que los registros hasta la mitad del siglo XX se centran en noticiarios e institucionales. Iniciada la segunda mitad del siglo, cuando una Europa destruida no ofrecía un panorama propicio para la creación, la presencia en el Cono Sur de los directores que en los treinta habían abogado por un documental militante en la Europa de entreguerras, permitió sembrar su semilla en un continente que aunque no había vivido la guerra, si era escenario sempiterno de tensiones sociales y políticas. De este modo el cine de la región, bajo el amparo del por entonces recién surgido movimiento del Nuevo Cine Latinoamericano, aprovecha el documental para retratar la lucha de unas masas que buscaban condiciones sociales más equitativas.

Pasados los años, el documental se traslada a la TV y se instala en algunos canales especializados, alcanzando mayor difusión (Rosique, 2015). Así, la introducción del video análogo y más tarde del digital, abrió campo a una cantera de temas y exploraciones que democratizaron el género, al multiplicarse el número de realizadores y la cantidad de temáticas tratadas. Al mismo tiempo, el abaratamiento de la producción y la asequibilidad cada vez mayor que permitió el video casero, hicieron posible que éste se convirtiera en recurso de expresión de muchas comunidades. Las historias marginales e imperfectas comenzaron a reclamar su derecho propio a existir, al tiempo que los procesos de formación de videógrafos en las comunidades, permitieron diversificar los discursos audiovisuales hasta ahora imperantes.

Al respecto de las tendencias en el modo de hacer documentales, se puede mencionar que hasta los años 80, las técnicas empleadas por el Cinema verité, consistentes en la intervención de la realidad para catalizar situaciones, son las que más se aproximan a un diálogo interactivo con los sujetos filmados, ausente en los otros modos documentales caracterizados por Nichols (1991). Pero no sería sino hasta la aparición del Internet, que esta interacción pasaría a tener un nuevo eje cartesiano, que la sustraería de su bidimensionalidad y la haría atravesar la pantalla, para interactuar ahora con los espectadores.

Con la entrada del milenio se inicia una nueva etapa en el ámbito de los medios y por ende del cine documental. Durante los primeros años de la década del 2000, la utilización de contenido multimedia como apoyo a las prácticas educativas, se hizo popular y proliferaron cursos y enciclopedias tipo Encarta, que a través del CD-ROM como medio, permitían la exploración por parte del usuario de los contenidos de su interés. Esta posibilidad de encadenamiento temático fue una herramienta de entrenamiento para entender más tarde las lógicas de la Web.

Desde la perspectiva del cine documental, la consolidación del Internet favoreció que hacia finales de la primera década del siglo XXI, comenzaran a surgir los primeros documentales para la Web, pero no sería sino hasta la aparición de los teléfonos inteligentes, que el tema haría explosión, permitiendo las exploraciones infinitas y los cruces de lenguajes a los que hoy nos avocamos. Si bien este es un proceso en desarrollo, lo cierto es que la ruptura de la cuarta pared y el encuentro con el usuario, ha resultado un ejercicio conveniente para creadores y públicos, en especial en cinematografías como la Latinoamericana, que han encontrado a través de proyectos colaborativos e interactivos, una oportunidad de visibilizar problemáticas marginalizadas.

\subsection{Mediamorfosis documental: experiencias de ida y vuelta entre el género y la tecnología.}

El fenómeno que se está produciendo en la actualidad, frente a la velocidad con la que avanza la tecnología y la aparición de nuevos gadgets, incide en el modo como las narrativas buscan acoplarse a los medios y viceversa. En este proceso atraviesan por lo que Altman (1999, citado en Gifreu-Castells, 2013), denomina intermedialidad: una especie de adolescencia en que la identidad de un medio entra en proceso de redefinición de sus propios recursos de lenguaje.

Para Gaudreault y Marion (2005), dos momentos se presentan cuando un nuevo medio surge: el primero relacionado con su aparición y el segundo con su consolidación. A esta teoría la denominan el doble nacimiento de los medios. Durante la fase de aparición, el medio evoca a otros para justificarse. Durante su emergencia, aparecen las características propias que lo distancian de sus predecesores. En la transición entre la primera y segunda etapa, el medio adquiere identidad propia, gracias a la insubordinación frente a las prácticas que le dieron legitimidad. Es entonces cuando se produce su segundo nacimiento.

Podría decirse que el documental contemporáneo se encuentra en fase de intermedialidad, buscando afianzar nuevos modelos narrativos, para romper las convenciones previamente acordadas por realizadores y teóricos del género. Los trabajos que se presentan a continuación, apropian recursos de otros lenguajes para transmitir su mensaje, y son un ejemplo de las transformaciones del cine documental, que desde su propia Mediamorfosis, se apronta a un segundo nacimiento.

Las experiencias documentales a las que se hará referencia, buscan ofrecer un panorama general sobre la diversidad 


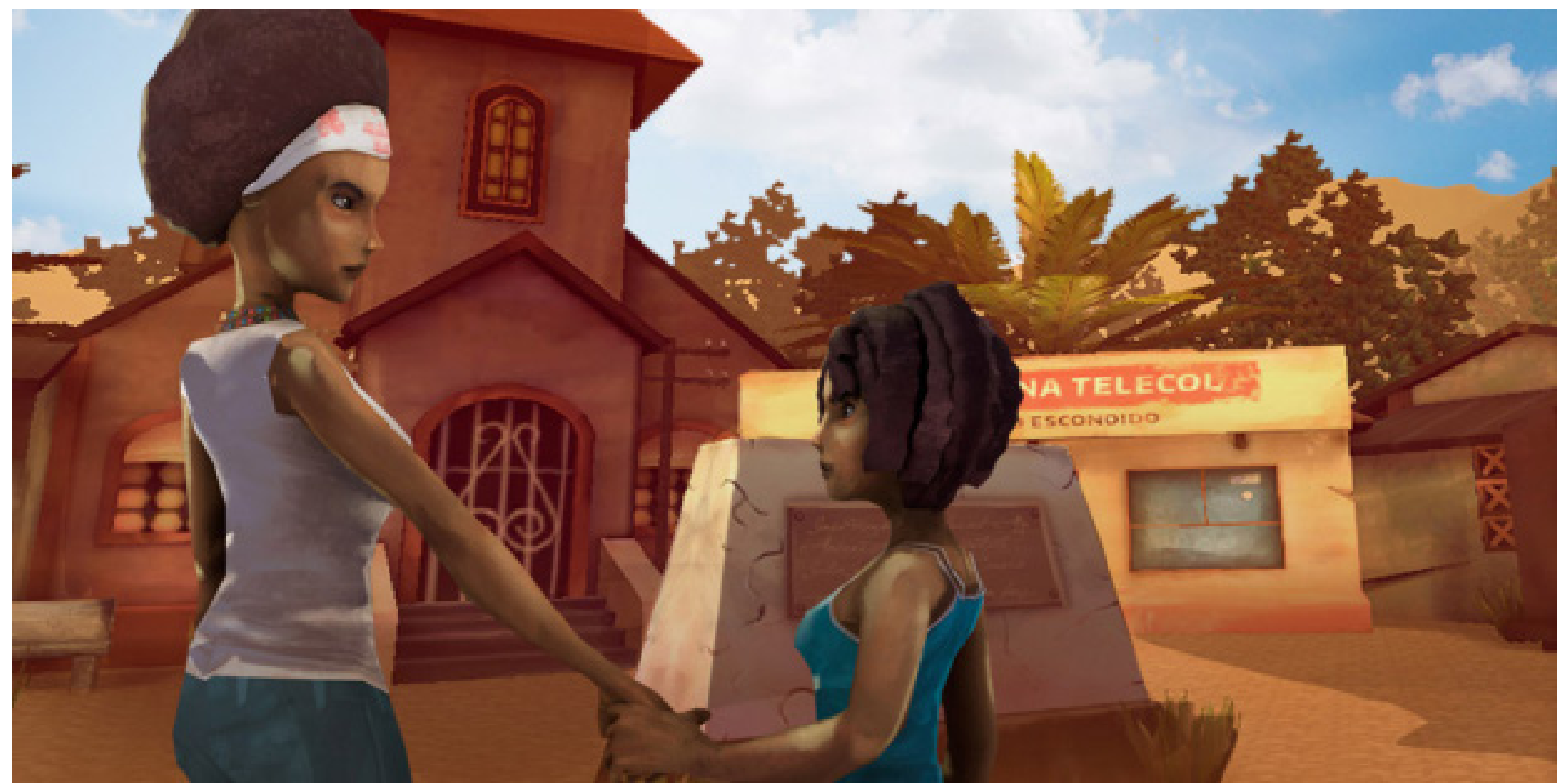

Figura 1. Imagen del video juego Reconstrucción, de Ayala y Triana (2017). Colombia. Fuente: www.reconstruccion.co/\#overview.

de abordajes presentes en la actualidad. Fueron elegidas en razón de la originalidad de su formato y los recursos audiovisuales, técnicos e interactivos que invocan. Se quiso reducir la muestra a documentales para la Web, documentales gamificados y experiencias inmersivas en realidad virtual, sin desconocer otras variables narrativas que existen hoy en día. Para la selección se tuvo en cuenta la cercanía con el contexto retratado, lo que permitió tomar en cuenta más allá de la obra, cuestiones relacionadas con su desarrollo y producción. Por este motivo la mayoría de los trabajos mencionados son colombianos. Finalmente, se consideró que dichos proyectos tuvieran una narrativa compleja, que se desarrollara progresivamente a través de la interacción, y que abordaran problemáticas sociales, por encima de ejercicios introspectivos o más inclinados hacia el goce estético.

\subsubsection{Documentales gamificados o juegos serios: Reconstrucción e Impaciente.}

La primera experiencia a la que se hará alusión en este recorrido hacia la comprensión de las transformaciones del género, es el documental gamificado Reconstrucción (2017) de Patricia Ayala y Álvaro Triana. El trabajo se realizó durante los diálogos de paz del gobierno de Colombia con la guerrilla de las FARC y refleja las esperanzas y expectativas que en ese momento se tenían frente al fin del conflicto armado. A través de la aplicación móvil, se puede explorar un relato de postguerra en que la protagonista, de quien el usuario asume su avatar, regresa al pueblo del que huyó por el hostigamiento de los actores armados, y rememora los acontecimientos que la llevaron a desplazarse a la ciudad, abandonándolo todo. El objetivo del juego es ofrecer una mirada del conflicto a través del relato del personaje principal y brindar una perspectiva de las consecuencias de la guerra sobre las víctimas. También busca involucrar al usuario al hacerlo partícipe de las decisiones que toman los personajes, que como mencionan los creadores, más que ser buenos o malos, son seres humanos que deben actuar en situaciones de gran presión.

Adicional a la interacción principal, el juego ofrece información contextual como mapas, videos y mini-infografías, que permiten conectar con historias reales de personas desplazadas por el conflicto armado. La lógica del reto-retribución, característica de los videojuegos, también se hace presente en determinadas escenas en las que el personaje se ve obligado, por ejemplo, a atravesar un campo minado. De este modo el trabajo no ofrece una perspectiva convencional de la guerra en Colombia, ni se limita a la lúdica, sino que hace vivir y comprender al usuario los sucesos dolorosos, a los que aún se enfrenta la población rural que se halla en medio de los viejos y nuevos actores armados.

En esa misma línea se encuentra Impaciente (2016) de Jorge Caballero, que hace parte del transmedia Paciente (2016), compuesto por un documental lineal, una serie de cortometrajes realizados por distintos cineastas colombianos, un libro web que recoge las historias recabadas durante la investigación y el mencionado juego.

El proyecto transmedia habla de las dificultades que tienen los ciudadanos para acceder a los servicios de salud en Colombia. Bajo el lema "juega y pierde el tiempo a costa de tu salud", el documental gamificado Impaciente, le propone al usuario distintas situaciones a través de micro relatos interactivos, que lo hacen atravesar la maraña burocrática e hilarante 


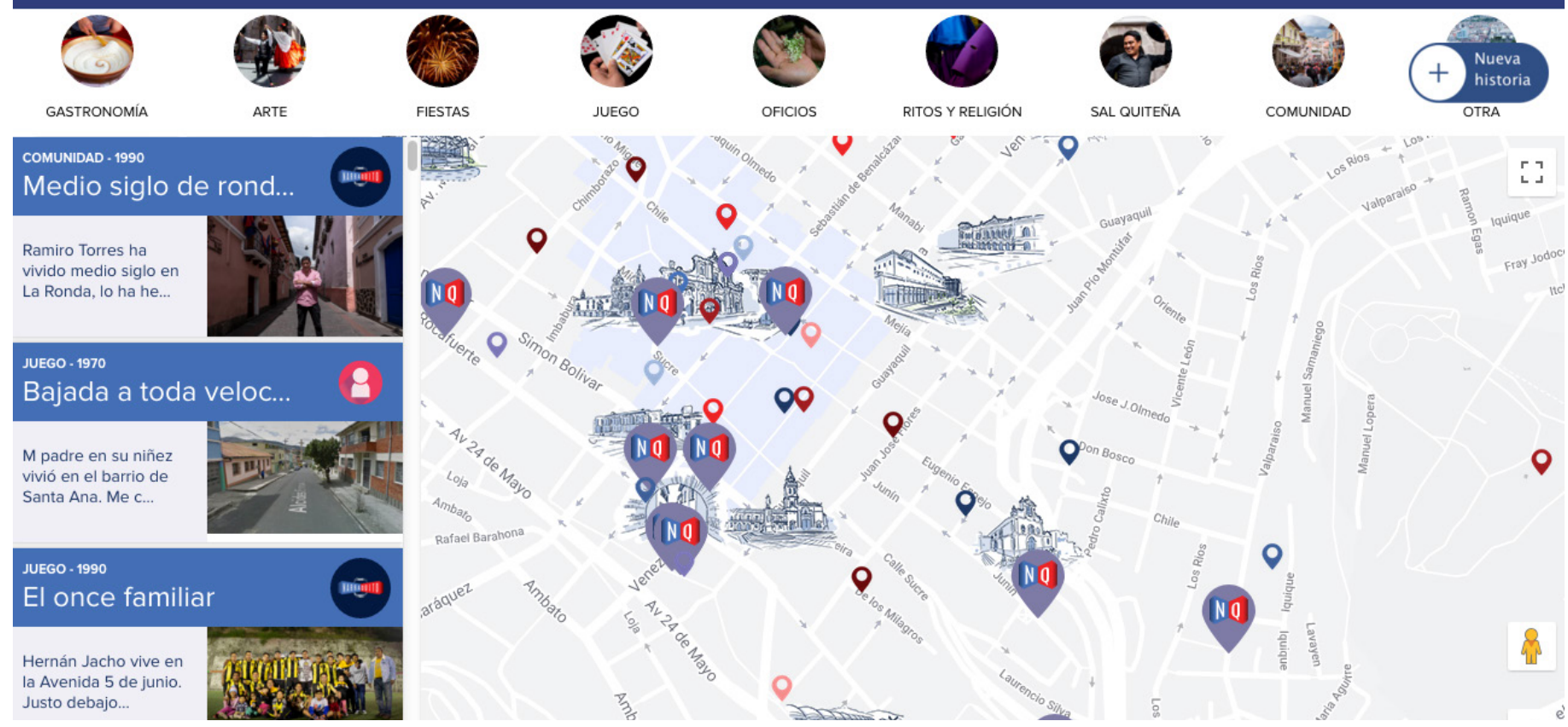

Figura 2. Interfaz del documental web NarraQ̣uito, de Urgilés, Arias y Maruri (2019). Ecuador. Fuente: www.narraquito.com

del sistema de salud. Para ello el usuario debe escanear un código QR, que sincronizará la pantalla del computador y la del celular. Mientras en el computador se le plantean situaciones y obstáculos, como solicitar citas con especialistas o exámenes de laboratorio, al teléfono le llegan mensajes que conectan con testimonios en video de pacientes que vivieron casos similares, e información contextual como notas de prensa relacionadas con el tema tratado. Al terminar la interacción nada se resuelve para quien está jugando, lo máximo que obtiene es llegar al final de cada historia habiendo perdido menos tiempo, es decir habiendo hecho menos interacciones en el proceso. Así, el dispositivo narrativo usado en Impaciente, apela a la lógica de lo absurdo al mejor estilo kafkiano, para que el usuario comprenda su propio desamparo.

\subsubsection{Documentales para la Web, dos experiencias cartográficas: NarraQuito y Pregoneros de Medellín.}

Dentro de los documentales Web considerados en este panorama, se juntan dos proyectos cartográficos que abordan de manera distinta la relación con el espacio y los usuarios. La primera a la que se hará mención es NarraQuito (2019) de Juan Pablo Urgilés, Angélica Arias y Mónica Maruri. Esta es una experiencia transmedia, interactiva y colaborativa, cuyo propósito es rescatar la memoria del centro histórico de Quito, a través de mapas, infografías, documentales interactivos e inmersivos, en los que el usuario puede profundizar sobre determinados temas relacionados con personajes, eventos y lugares característicos del centro de la ciudad, y al mismo tiempo hacer aportes a la construcción de la memoria colec- tiva en torno a dicho espacio.

La navegación es realizada a través de un mapa que se halla en la web, en el que el usuario encuentra las historias producidas por el equipo de realización (documentales interactivos y en $360^{\circ}$ ), pero en el que también se ubican las anécdotas que los usuarios van subiendo a la red y que aparecen marcadas dentro del mismo mapa. Estas anécdotas constituidas por recuerdos familiares en torno a celebraciones, negocios tradicionales, fotos antiguas y pequeños videos, van conformando el gran álbum familiar del centro de la ciudad y sus vecinos. La retribución para los participantes son bonos de descuento en negocios del sector, de esta manera se traslada la experiencia del espacio virtual al real. Así las cosas, lo que hace sumamente interesante el diseño de NarraQuito, es la puesta en valor de los relatos mínimos, para la construcción a gran escala del proyecto.

Otra experiencia que apela a la cartografía virtual es Pregoneros de Medellín (2015) de Angela Carabalí y Thibault Durand. El proyecto recoge las historias de un grupo de vendedores ambulantes del centro de la ciudad, y registra cómo cada uno de ellos tiene un pregón particular a la hora de promover sus productos. El proyecto transmedia se compone del documental interactivo para la web, unas cápsulas documentales para la TV y una exposición fotográfica. A las historias se accede recorriendo las calles como un transeúnte más, mediante el botón de scroll del mouse y encontrando a cada uno de los pregoneros dentro de la multitud. Una vez identificados se puede conocer un fragmento de su vida en un micro documental lineal. A medida que se profundiza en la exploración del espacio, se puede acceder a nueva información de los per- 




Figura 3. Metaverso VR, realidad virtual, de García, Pistolesi, Cuevas y López (2017). España. Fuente : archivo personal.

sonajes retratados, cuando son ubicados nuevamente en una calle distinta. Adicional a las historias de vida que hablan del país de la supervivencia, el gran aporte que hace el trabajo es el registro sonoro del centro de la ciudad, que le permite al usuario una inmersión sensorial a través del sonido posicionado. Los pregones, compuestos por canciones y dicharachos graciosos, son la memoria a futuro de una ciudad que se transforma aceleradamente y de unos oficios que pronto pueden desaparecer.

\subsubsection{Experiencias inmersivas en realidad virtual: Metaverso y Entre Luces y Sombras.}

Entrando en el tema de las narrativas inmersivas VR, se hará referencia en primer lugar a Metaverso (2017), producción de los estudiantes del Máster en Teoría y práctica del documental creativo de la Universidad Autónoma de Barcelona. Bajo la premisa ¿Está preparado el ser humano para ser amo de su propia evolución?, Metaverso propone al usuario sumergirse en un viaje a través de la interfaz de la supercomputadora MareNostrum, aparato ubicado en el Barcelona Supercomputing Center, y que dedica parte de su capacidad al análisis del genoma humano.

Durante el recorrido el usuario tiene la oportunidad de informarse sobre los alcances de la modificación genética en el hombre, y participar en una simulación interactiva en la que debe elegir entre una serie de derroteros deseables como la inmortalidad, la inteligencia superior, o la belleza, para decidir el futuro de la humanidad. Cada elección está repre- sentada por referencias de la filosofía, la literatura universal, la historia del arte y la cultura popular, que demuestran que la inmortalidad y la superioridad física e intelectual, han hecho parte de los anhelos del hombre durante siglos. La elección en cada caso conducirá al usuario a observar un futuro hipotético, que es consecuencia directa de sus decisiones: a quien elige la inmortalidad, lo espera un mundo sin recursos naturales, o a quien elige el raciocinio por encima de la intuición, lo esperará una sociedad autómata y sin emociones. El árbol de interactividad permite que en cada visionado se puedan hacer elecciones distintas, obteniendo un resultado diferente cada vez. Al final de la experiencia la sensación no es agradable, el usuario como centro del relato es causa y efecto del desastre al que se abocaría la humanidad, si no se reglamenta la experimentación genética. En este sentido el trabajo es un llamado a asumir críticamente las fantasías tecno-científicas de la era moderna.

Siguiendo con los relatos inmersivos en realidad virtual, se hará mención a Entre Luces y Sombras (2020). La experiencia hace parte de un transmedia en desarrollo, que cuenta la historia del primer cine hecho en Colombia, a través de la figura del pionero Félix Joaquín Rodríguez. El VR está compuesto por cuatro capítulos, que recogen distintos momentos de la vida del personaje, poniéndolos en contexto con la historia del cine y de Colombia. La experiencia le propone al usuario tomar el lugar del cineasta y ver a través de sus ojos, mientras es guiado por la voz de su musa Isabel. En el Capítulo III por ejemplo, el usuario debe ubicar mediante el sistema de point and click, a los personajes principales de la película original de don 


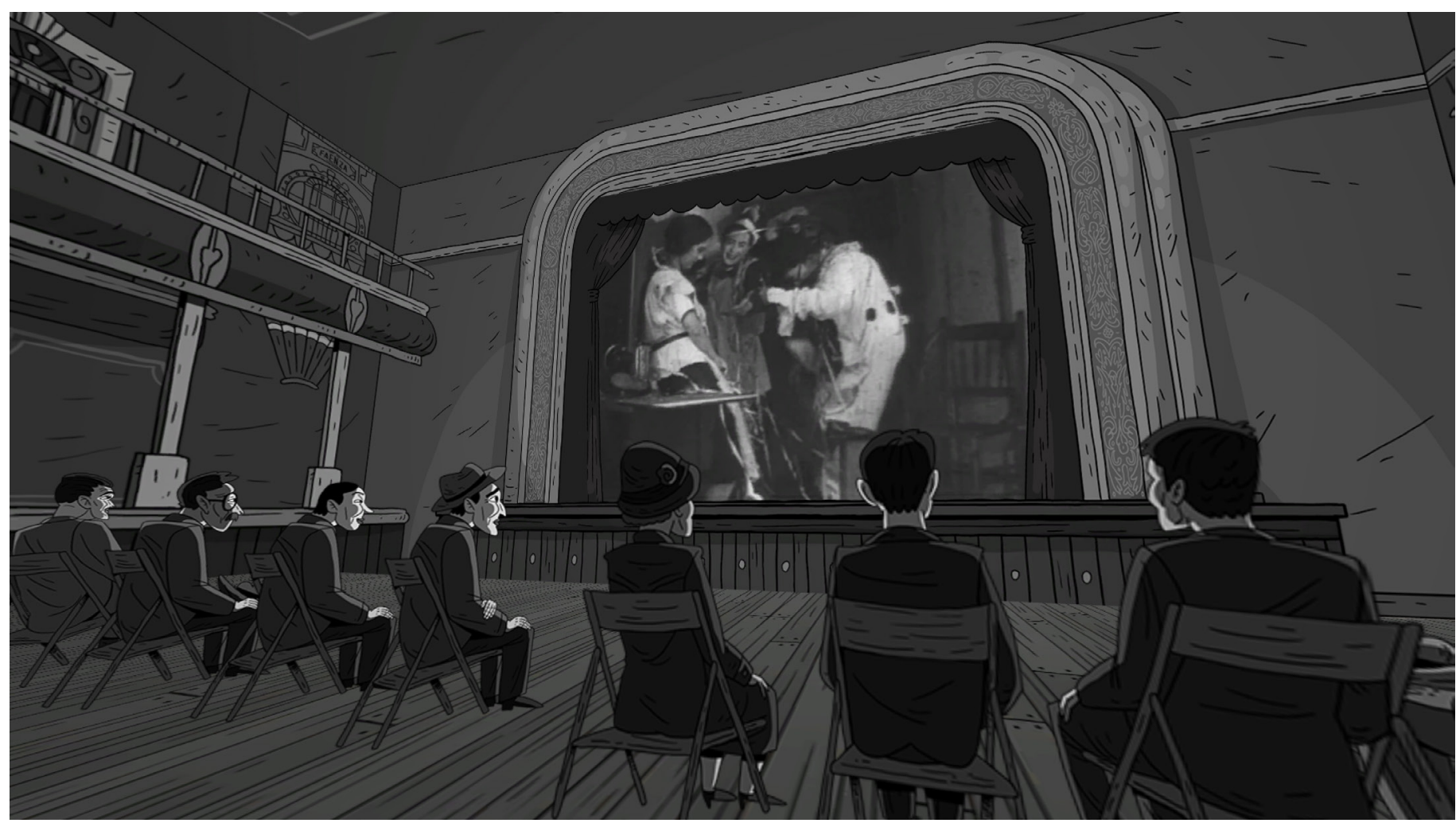

Figura 4. Entre Luces y Sombras, realidad virtual, de López (2020). Colombia. Fuente: archivo personal.

Félix, Llamada Alma Provinciana (1926). A medida que encuentra al terrateniente, al estudiante, al campesino y a la obrera, la cámara de cine que el usuario tiene frente suyo, se acciona y de este modo emula filmarlos.

Cuando logra su cometido, el usuario se traslada al Teatro Faenza, lugar donde originalmente se estrenó la película en los años veinte y ve un fragmento de la cinta real en la pantalla. Al final del recorrido el usuario no solo habrá repasado una página importante de la historia nacional, caracterizada por los conflictos sociales y la lucha obrera, sino que habrá experimentado el haber rodado la película más representativa del periodo silente colombiano. La inmersión en este caso es total, cuando se logra combinar presencia e interactividad, a través del recurso de poner la primera persona del relato, en cabeza del usuario.

\section{Conclusiones}

La observación que se recoge tras analizar los ejemplos precedentes, es que las nuevas formas de narrar que se incorporan en el panorama actual de medios, buscan desde las más variadas posturas, un espacio de existencia por encima de las convenciones ya aceptadas y las fronteras de género previamente establecidas en el cine documental. Se podría decir que gracias a estos ejercicios, el documental se halla en una etapa de intermedialidad y se aproximaría a su segundo nacimiento.

Más allá de cualquier desarrollo tecnológico, el elemento diferencial entre los viejos y los nuevos relatos, es la presencia de unas audiencias activas, capaces de involucrarse en las historias y operar bajo sus lógicas promoviendo y generando contenido. Este esquema equilibra la balanza histórica que hacía unidireccional el discurso, y permite diversificar miradas frente a los temas abordados.

Aunque no en todos los casos la participación toma la forma de producción de contenidos, la posibilidad de interactuar con el relato y las opciones ofrecidas al usuario en la exploración, le permiten una relación más activa con el medio y las historias, lo que termina siendo fundamental a la hora de conectar con las nuevas audiencias y el público más joven.

Resulta un elemento relativamente común en la mayoría de experiencias reseñadas, la producción de efectos encaminados a dirigir la atención del usuario, buscando que se involucre personalmente en la trama. Ya sea acompañando las desventuras de una víctima del conflicto armado, perdiendo la paciencia mientras espera acceder al sistema de salud, decidiendo el futuro de la humanidad o filmando una película, el usuario asume roles que lo identifican y lo llevan a experimentar sensaciones. El mensaje ya no se transmite como mera información, sino que en el nuevo panorama mediático, entra a operar también en el nivel emocional.

Pese a haber pasado casi un siglo desde la aparición de Nanook el esquimal (1922), la Mediamorfosis del cine documental, en el escenario de la convergencia y el ecosistema de medios, acerca cada vez más el género a su esencia fundacional: La postura empática. El encuentro con el otro se hace real en las nuevas formas de consumo, apropiación y producción 
de contenido, apreciables en los documentales Web, gamificados y las experiencias VR aquí descritas. Esta nueva relación entre realizador, sujetos filmados y espectadores, hace posible lo que el buen Robert Flaherty, padre fundador del género, profetizara como su misión: la "de promover la mutua comprensión entre los pueblos" (Flaherty, 1989, p. 151).

\section{Bibliografía}

Acin, R. (productor) y Buñuel, L. (director). (1933). Las Hurdes tierra sin pan [documental]. España: Ramón Acín productor.

Ayala, P. y Triana, A. (directores). (2017). Reconstrucción [documental gamificado]. Colombia: Pathos Audiovisual.

Altman, R. (2000). Los géneros cinematográficos. Paidós.

Barnouw, E. (2006). El documental, historia y estilo. Gedisa.

Caballero, J. (director). (2016). Impaciente [documental web gamificado]. Colombia: Gusano films.

Carabali, A. y Durand, T. (directores). (2015). Pregoneros de MedeLlin [documental web]. Colombia: Carabali-Thibault Durand.

Capra, F., Litvak, A. y Veiller, A. (directores). (1942-1945). Wy We Fight [serie documental]. Estados Unidos: Oficina de Información de Guerra.

Fidler, R. (1998). Mediamorfosis. Comprender los nuevos medios. Granica.

Flaherty, R. (director). (1922). Nanook from the north [documental]. Estados Unidos: Révillon Frères-Pathé Exchange.

Flaherty, R. (1989). Los postulados del documental. En Romaguera, J. y Alsina, H. (Eds), Textos y Manifiestos del cine, (pp. 151-154). Cátedra.

García, J., Pistolesi, G., Cuevas, J. y López, N. (directores). (2017). Metaverso VR [realidad virtual]. España: Universidad Autónoma de Barcelona.

Gaudreault, A. y Marion, P. (2005). A medium is always born twice. En Early Popular Visual Culture, 3(1), 3-15. https://doi. org/10.1080/17460650500056964

Gifreu-Castells, A. (2013). El documental interactivo como nuevo género audiovisual. Estudio de la aparición del nuevo género, aproximación a su definición y propuesta de taxonomía y de un modelo de análisis a efectos de evaluación, diseño y producción. (Tesis doctoral). http://agifreu.com/interactive_documentary/TesisArnauGifreu2012. pdf

Granada, N. (productor) y López, N. (director). (2020). Entre Luces y Sombras [realidad virtual]. Colombia.

Irigaray, F. (2015). Periodismo Transmedia: Nuevas posibilidades narrativas y de experiencia de usuario. En Roitberg, G. y Piccato, F. (comps.), Periodismo disruptivo. Dilemas y estrategias para la innovación, (pp. 167-172). La Crujía.

Jenkins, H. (2008). Convergence culture. La cultura de la convergencia en los medios de comunicación. Paidós.

Logan, R. (2010). Understanding new media: Extending Marshall McLuhan. Peter Lang.

Lovato, A. (2015). Del periodismo multimedia al periodismo transmedia. En Irigaray, F. y Lovato, A. (Eds), Producciones Transmedia de no ficción, análisis experiencias y tecnologías (pp. 33-51). UNR Editora.

Morin, E. y Rouch, J. (directores). (2015). Chronique d'un été [documental]. Francia: Argos Films.

Nichols, B. (1991). La representación de la realidad. Paidós.

Paranaguá, P. (2003). Cine documental en América Latina. Cátedra.

Rabiger, M. (2001). Dirección de Documentales. Instituto de Radio y Televisión Española.

Rosique, G. (2015). Presencia e implantación del documental interactivo en los programas de estudio de las universidades españolas. Obra Digital, (8), 62-82. https://doi.org/10.25029/od.2015.53.8

Valdettaro, S. (2015). El transmedia: una aproximación a lo maravilloso-hipermoderno. En Irigaray, F. y Lovato, A. (Eds), Producciones Transmedia de no ficción, análisis experiencias y tecnologías, (pp. 62-77). UNR Editora.

Urgilés, J., Arias, A. y Maruri, M. (directores). (2019). NarraQuito [documental web]. Ecuador: Imán transmedia e Instituto Iberoamericano del Patrimonio Natural y Cultural IPANC.

Vigo, J. (director). (1929). À propos de Nice [documental]. Francia: Gaumont-Franc-Film-Aubert.

Vigo, J. (1989). El punto de vista en el documental "A propósito de Niza". En Romaguera, J. y Alsina, H. (Eds), Textos y Manifiestos del cine, (pp.134-138). Cátedra.

\section{CV}

Nazly López Díaz. Politóloga egresada de la Universidad Nacional de Colombia (2004) y documentalista de la Escuela Internacional de Cine y TV de San Antonio de los Baños, Cuba (2008). Máster en Teoría y práctica del Documental Creativo de la Universidad Autónoma de Barcelona (2017). Maestranda en Comunicación digital interactiva de la Universidad Nacional de Rosario, Argentina (2019-). Docente de cine y asesora de documentales de corto y largometraje en Colombia, Cuba y Panamá. Investigador Junior Minciencias Colombia.

\section{осм Observatorio deCibermedios}

https://observatoriocibermedios.upf.edu/

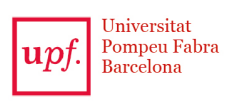

Departamento de Comunicación
Grupo DigiDoc

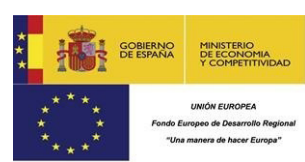

El Observatorio de Cibermedios es una producción del Grupo de Investigación en Documentación Digital y Comunicación Interactiva (DigiDoc) del Departamento de Comunicación de la Universitat Pompeu Fabra.

El Observatorio de Cibermedios (OCM) forma parte del proyecto del Plan Nacional "Narración interactiva y visibilidad digital en el documental interactivo y el periodismo estructurado". RTI2018-095714-B-C21 (MINECO/FEDER), Ministerio de Ciencia, Innovación y Universidades (España). 